\title{
MODÉLISATION NUMÉRIQUE DES FACETTES TRIANGULAIRES
}

\author{
Julien Seguinot
}

3 juillet 2007

Sous la direction de Carole Petit et Bertrand Meyer.

\section{Table des matières}

1 Formation des facettes triangulaires 3

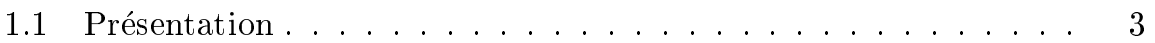

1.2 Variabilité . . . . . . . . . . . . . . . 4

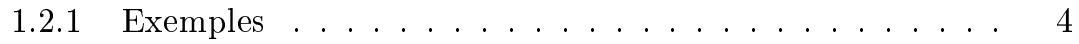

1.2.2 Paramètres tectoniques $\ldots \ldots \ldots \ldots \ldots$

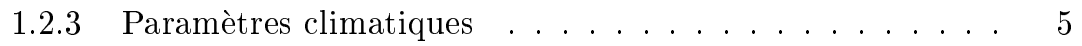

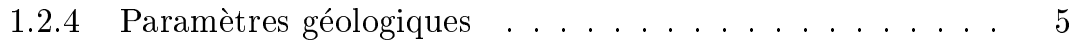

1.3 Problème inverse . . . . . . . . . . . . . . . 6

2 Modélisation 6

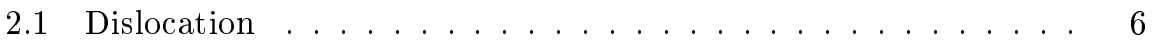

2.1.1 Position du problème . . . . . . . . . . . . 6

2.1 .2 Mise en équation . . . . . . . . . . . . . . 7

2.1 .3 Discrétisation . . . . . . . . . . . . . 7

2.2 Érosion diffusion $\ldots \ldots \ldots \ldots \ldots \ldots \ldots$

2.2 .1 Position du problème . . . . . . . . . . . 8

2.2 .2 Mise en équation . . . . . . . . . . . . . 8

2.2 .3 Discrétisation . . . . . . . . . . . . . . . 9

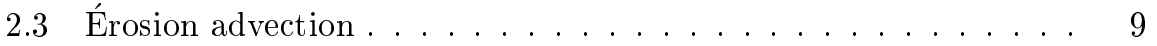

2.3.1 Position du problème . . . . . . . . . . . . . . . 9

2.3 .2 Mise en équation . . . . . . . . . . . . . . . . 9

2.3 .3 Discrétisation . . . . . . . . . . . . . . . . 11

3 Résultats 11

3.1 Paramètres d'érosion . . . . . . . . . . . . . . . . 11

3.2 Notations . . . . . . . . . . . . . . . . . 12

3.3 Domaine de formation des facettes triangulaires . . . . . . . . . 13

3.4 Plan $k_{e}=$ cte . . . . . . . . . . . . . . . 15

3.5 Variations du nombre caractéristique d'érosion . . . . . . . 15 
4 Application à la faille de Sparte $\quad 16$

4.1 Situation . . . . . . . . . . . . . . . . 16

4.2 Vallées en verre à vin . . . . . . . . . . . . . . . . 17

4.3 Ruptures de pentes . . . . . . . . . . . . . . . . . 17

\section{Résumé}

Ce travail a été réalisé dans le cadre d'un stage d'un mois à la fin de mon année de license trois au département terre-atmosphère-océan de l'école normale supérieure de Paris. J'ai effectué ce stage du 29 mai au 2 juillet 2007 au laboratoire de tectonique de l'université Pierre et Marie Curie de Paris sous la direction de Carole Petit et de Bertrand Meyer.

La modélisation numérique de la formation de facettes triangulaires sur un escarpement de faille normale est une étude qui, à ma connaissance, n'a pas encore été menée dans le monde de la recherche.

Mon travail a consisté en la prise en main d'un modèle numérique de facettes triangulaires écrit par Carole Petit sous Matlab, la modification de deux parties de ce programme (érosion par diffusion et par advection,) la réalisation de nombreux tests sur quelques paramètres de ce programme, et la réflexion sur une application possible du modèle au cas de la faille de Sparte.

\section{Abstract}

This work come within the scope of a one-month-long training period at the end of my bachelor third year at the terre-atmosphère-océan department of the école normale supérieure in Paris. I worked from May 29th to July 2nd, 2007 at the tectonics laboratory of the Pierre et Marie Curie university in Paris, under the supervision of Carole Petit and Bertrand Meyer.

Numerical modeling of the formation of triangular facets on a normal fault scarp is, to my knowledge, a research subject which was not undertaken up to now.

My work consisted of getting used to a triangular facets model written by Carole Petit under Matlab. I modified two parts of this model to account for diffusive and advective erosion. The program was tested under varying erosion parameters. The possible application of the developped model to the Sparta fault case has been investigated. 


\section{Formation des facettes triangulaires}

\subsection{Présentation}

Les facettes triangulaires sont une morphologie caractéristique des reliefs extensifs [2]. Elles se forment le long de failles normales, en général dans les zones de rifting. Leur origine est à la fois tectonique et climatique.

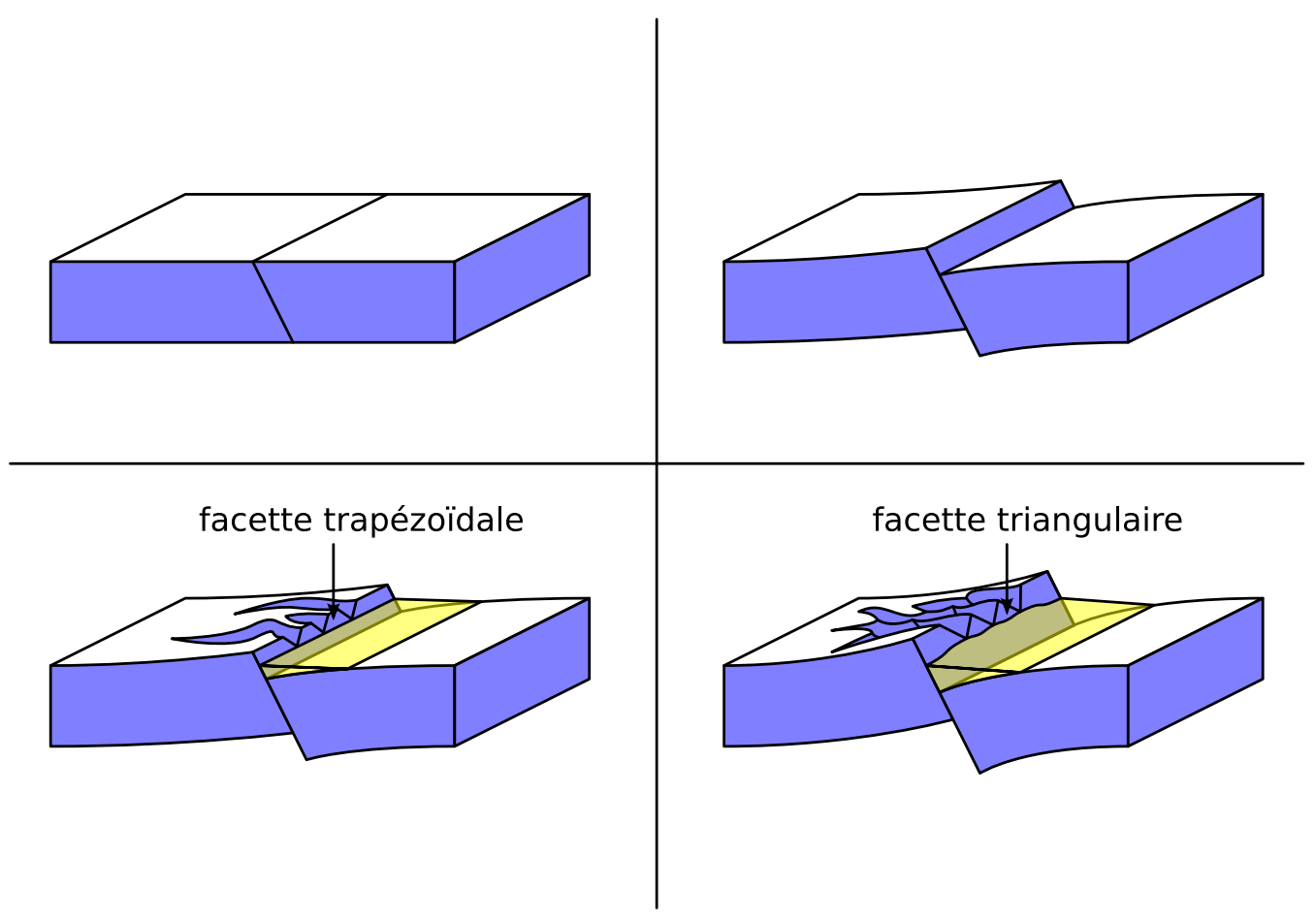

FIG. 1 - Formation des facettes triangulaires.

Lors du jeu d'une faille normale, le bloc remonté est proéminent et donc fort exposé à l'érosion (fig. 1.) Naissent alors des rivières qui incisent l'escarpement de faille et forment des vallées en $\mathrm{V}$. Avec l'augmentation du rejet de faille, ces vallées se creusent jusqu'à délimiter des crêtes et faire apparaître des facettes triangulaires sur l'escarpement. Ces facettes peuvent mesurer jusqu'à plusieurs centaines de mètres de haut.

Les facettes sont généralement accompagnées d'autres reliefs caractéristiques (fig. 2 :)

- bassin sédimentaire sur le bloc descendu ou mur;

- cônes alluviaux en bas des vallées; 
- Une ligne de partage des eaux qui délimite un bassin versant drainant dans le sens du pendages de la faille et un autre dans le sens opposé. Ceci est lié à la flexure lors du jeu de la faille.

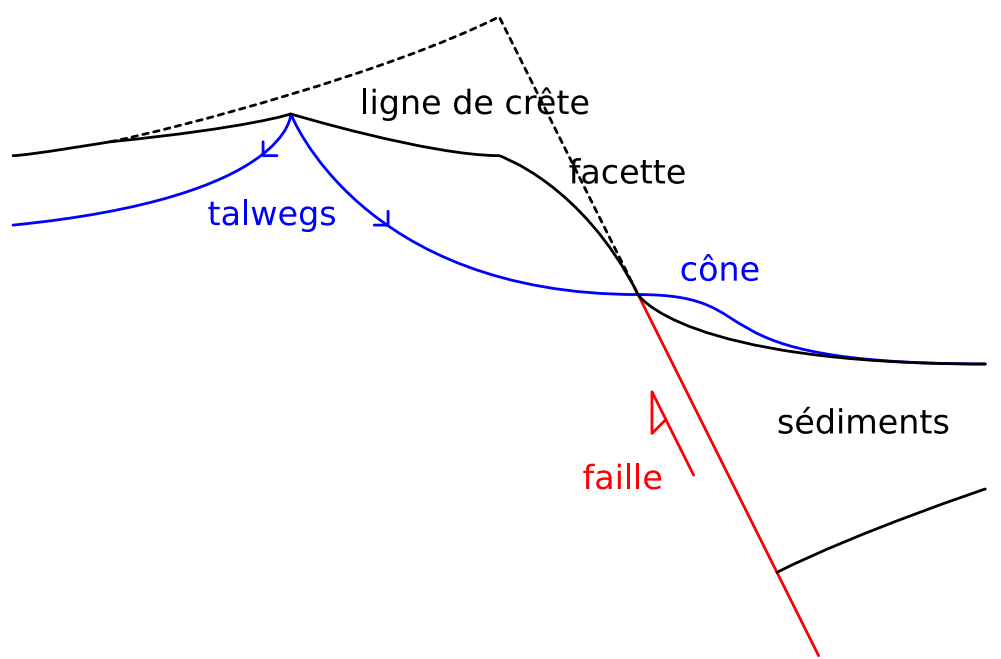

FIG. 2 - Coupe perpendiculaire à la trace de la faille.

\subsection{Variabilité}

\subsubsection{Exemples}

Tous les escarpements de failles normales actives ne présentent pas nécessairement de facettes triangulaires; et lorsqu'elles sont visibles, ces dernières ont des formes diverses, comme le montrent les trois exemples suivants.

La faille de Wasatch, à Salt Lake City (fig. 3) présente des facettes triangulaires très anguleuses au sein de grandes facettes trapézoïdales plus émoussées et délimitées par de larges vallées.

Les graben du point triple de l'Afar (fig. 4) présentent des escarpements de failles très peu érodés. Quelques courtes rivières délimitent de très larges facettes trapézoïdales. Noter les cônes de déjection. On distingue aussi quelques facettes triangulaires très pentues.

La vallée de Tunka dans le bassin en pull-apart du lac Baïkal (fig. 5) montre de nombreuses facettes triangulaires au formes très régulières. Noter les différentes générations de facettes imbriquées.

Quelles sont les origines de ces variations de morphologie? 


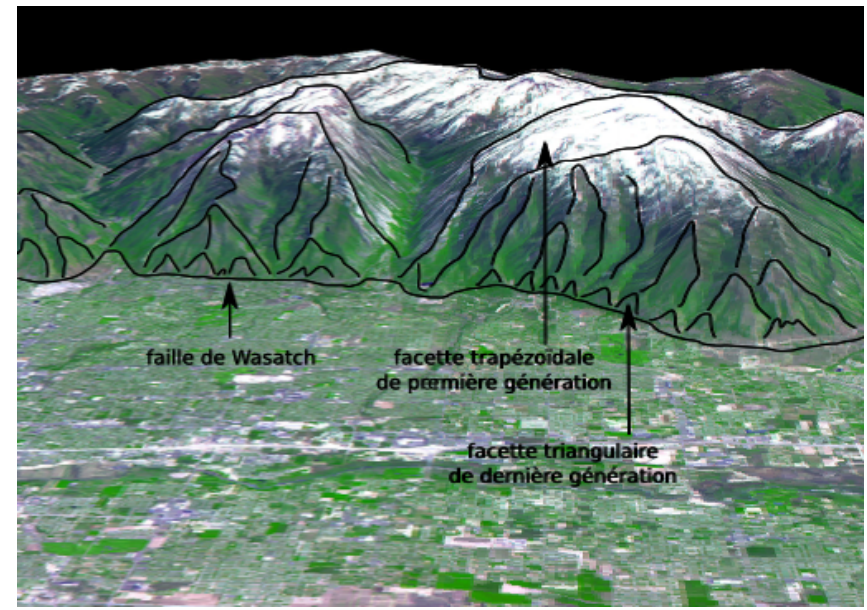

FIG. 3 - Faille de Wasatch à Salt Lake City - NASA Visible Earth [7].

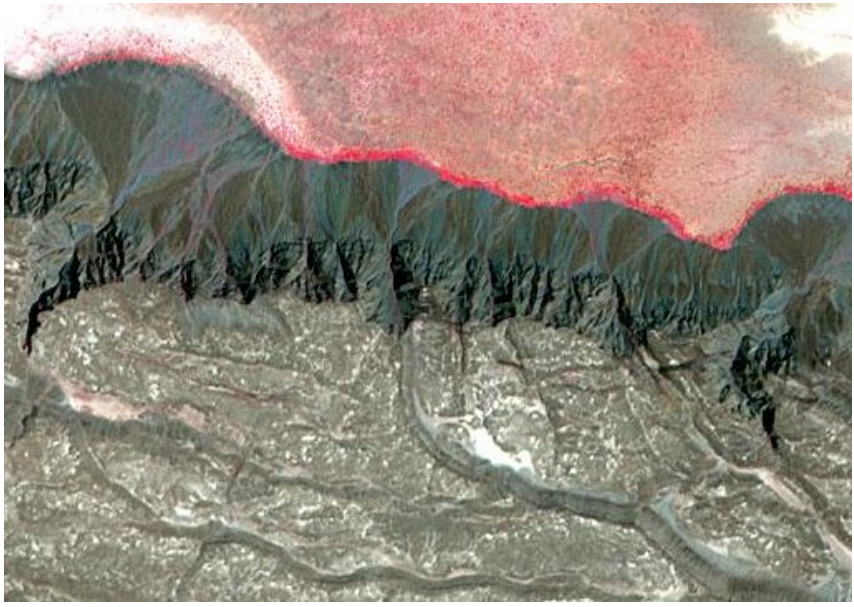

FIG. 4 - Rift dans la dépression de l'Afar. Noter les cônes de déjection — NASA Visible Earth [7].

\subsubsection{Paramètres tectoniques}

La vitesse de glissement sur la faille, son âge ainsi que son pendage vont contrôler la pente initiale du miroir de faille, la vitesse à laquelle celui-ci est exhumé et le temps pendant lequel il est soumis à l'érosion.

\subsubsection{Paramètres climatiques}

De plus, le climat local façonne l'escarpement de faille par érosion. Le paramètre dominant est la pluviométrie, mais l'érosion glaciaire peut parfois intervenir, comme dans le cas du lac Baïkal. Selon l'aridité ou l'humidité du climat, les phénomènes de ruissellement ou d'altération in situ vont prédominer et donner une morphologie différente à l'escarpement.

$-j_{d}$ : flux horizontal de matière en mètres carrés par an;

$-k_{d}$ : coefficient de diffusion en mètres carrés par an, qui diffère selon la nature du matériau (socle ou sédiment);

- $h$ : altitude du sol, fonction de $t, x$ et $y$;

- $t$ : temps en années depuis le début du fonctionnement de la faille;

- $x$ et $y$ : coordonnées horizontales en mètres dans un repère orthonormé;

\subsubsection{Paramètres géologiques}

Enfin, la forme des reliefs dépend de l'histoire géologique de la région. La réponse du substratum à l'érosion est différente selon la lithologie. De plus, la présence d'un drainage antécédent lié à une histoire géologique passée peut influencer la façon dont les facettes s'initient. 

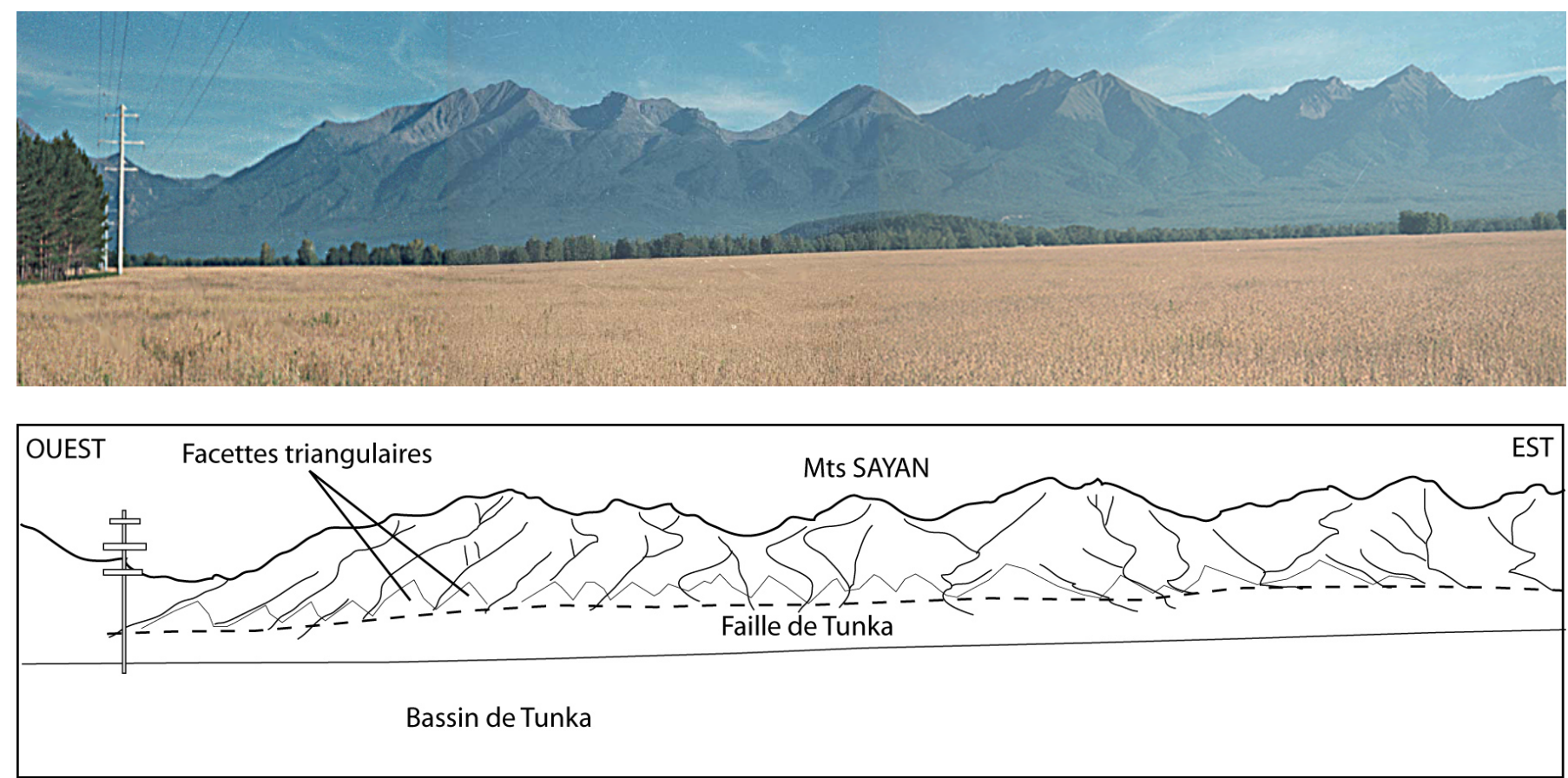

Fig. 5 - Vallée de Tunka près du lac Baikal - photo et dessin de Carole Petit.

\subsection{Problème inverse}

En général, la lithologie est connue ou peut être connue. En revanche, il est plus difficile de connaître l'activité passée de la faille ou l'histoire climatique de la région. La réalisation d'un modèle numérique peut donc se justifier par le fait qu'un tel modèle peut permettre, une fois calibré, d'estimer les paléoclimats d'une région connaissant sa tectonique, ou inversement, à partir de la topographie actuelle d'un escarpement de faille normale.

\section{Modélisation}

\subsection{Dislocation}

\subsubsection{Position du problème}

Soit une faille normale rectangulaire finie dont on ne considère que la partie centrale : on ne tient pas compte des effets aux extrémités. Cette faille est définie par sa longueur, sa largeur, son pendage, la fréquence et le glissement des séismes associés. Elle est incluse dans un milieu élastique dont la rhéologie est quantifiée par son module d'Young $E$ et son coefficient de Poisson $\nu$. 


\subsubsection{Mise en équation}

Le mouvement de la faille normale est calculé à l'aide du modèle de dislocation de Yoshimitsu Okada [4]. Ce modèle utilise la théorie de l'élasticité et détermine le champ des déplacements à la surface à partir du mouvement sur le plan de faille.

\subsubsection{Discrétisation}

La dislocation est appliquée à chaque fois qu'un séisme a lieu. À chaque séisme, la grille est donc modifiée et chaque point reçoit de nouvelles coordonnées.

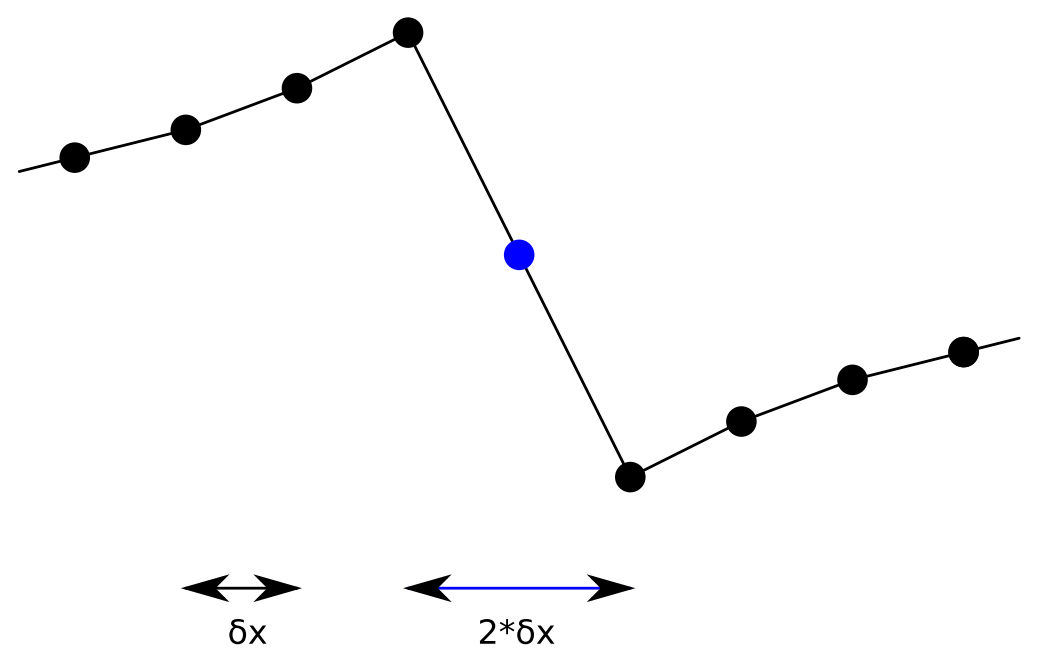

FIG. 6 - Remaillage.

Cela pose un problème au niveau du nœud de faille où le déplacement horizontal d'un bloc par rapport à l'autre provoque un élargissement de la maille. Cela signifie que perpendiculairement à la faille, la forme des facettes sera seulement décrite par deux points, l'un au sommet et l'autre à la base de la facette. On doit donc procéder régulièrement à un remaillage (fig. 6.) C'est à dire que lorsque la maille devient trop grande, on ajoute un point en son milieu. Dans les tests suivants, l'interpolation utilisée est linéaire et le remaillage est effectué à chaque fois que la maille atteint le double de sa taille originelle. 


\section{2 Érosion diffusion}

\subsubsection{Position du problème}

L'érosion par diffusion regroupe l'ensemble des déplacements désordonnés de matière. Il s'agit de la saltation grain par grain, des éboulements et des glissements de terrain. Ces phénomènes peuvent être interprétés comme le résultat de l'érosion éolienne mais aussi et surtout de l'altération des roches par les eaux de pluies. L'érosion diffusion est fortement liée à la quantité de précipitation.
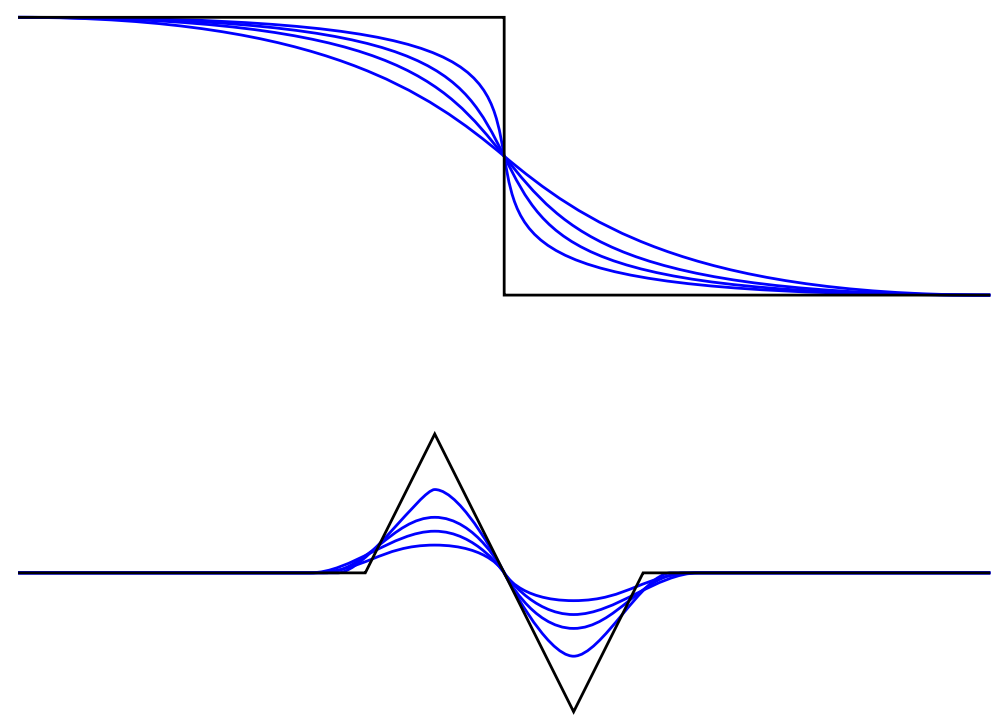

Fig. 7 - Effets de l'érosion diffusion.

Elle arrondit les angles, érode les crêtes et comble les bassins (fig. 7.) On distinguera ensuite le matériau d'origine (socle) du matériau déposé (sédiment.)

\subsubsection{Mise en équation}

Le flux de matière s'effectue dans le sens de la pente et est proportionnel à celle-ci :

$$
\overrightarrow{j_{d}}=-k_{d} \cdot \operatorname{grad}(h)
$$

- $j_{d}$ : flux horizontal de matière en mètres carrés par an;

$-k_{d}$ : coefficient de diffusion en mètres carrés par an, qui diffère selon la nature du matériau (socle ou sédiment);

- $h$ : altitude du sol, fonction de $t, x$ et $y$;

- $t$ : temps en années depuis le début du fonctionnement de la faille;

- $x$ et $y$ : coordonnées horizontales en mètres dans un repère orthonormé; 
Par conservation de la matière :

$$
\frac{\partial h}{\partial t}=\operatorname{div}\left(j_{d}\right)
$$

D'où l'équation de diffusion :

$$
\frac{\partial h}{\partial t}=k_{d} \cdot \triangle h
$$

Une telle équation modélise à la fois érosion et sédimentation.

\subsubsection{Discrétisation}

On utilise une méthode explicite du premier ordre. La condition aux limites choisie est l'annulation des flux de matière aux bords de la grille considérée.

Afin d'éviter des fuites ou créations de matière au niveau des interfaces socle / sédiment, on calcule d'abord l'ensemble des flux de matière sur toute la grille, puis la variation de topographie.

La méthode explicite n'est pas exacte et peut diverger si :

$$
k_{d} \cdot \frac{\delta t}{\delta x^{2}}>\frac{1}{2}
$$

$-\delta t:$ pas de temps;

$-\delta x$ : taille de la maille.

\section{3 Érosion advection}

\subsubsection{Position du problème}

L'érosion par advection regroupe l'ensemble des déplacements de matière organisés. Il s'agit de l'érosion, du transport et du dépôt de particules par les rivières, ou encore par les glaciers. Ces derniers ne sont pas pris en compte dans ce modèle. L'érosion par advection est principalement liée à la quantité de précipitation.

Elle creuse des chenaux, érode le fond des vallées et dépose sur les replats. On distinguera ensuite le matériau d'origine (socle) du matériau déposé (sédiment.)

\subsubsection{Mise en équation}

Soit un cours d'eau de largeur $w$, qui s'écoule le long de la courbe décrite par une abscisse curviligne $l$ (fig. 9.) Cette courbe n'est autre qu'une ligne de plus grande pente. On cherche à connaître les quantités d'eau et de sédiment que la rivière transporte. On note :

$-h$ l'altitude du sol, fonction de $t$, et $l$;

- $j_{e}$ le flux surfacique d'eau en $\mathrm{m}^{2} / \mathrm{an}$ dans le sens de l'écoulement;

- $j_{s}$ le flux surfacique de sédiments en $\mathrm{m}^{2} /$ an dans le sens de l'écoulement;

- $p$ les précipitations moyennes efficaces en $\mathrm{m} / \mathrm{an}$, c'est à dire l'eau de pluie qui n'est pas immédiatement évaporée ni absorbée par le sol; 


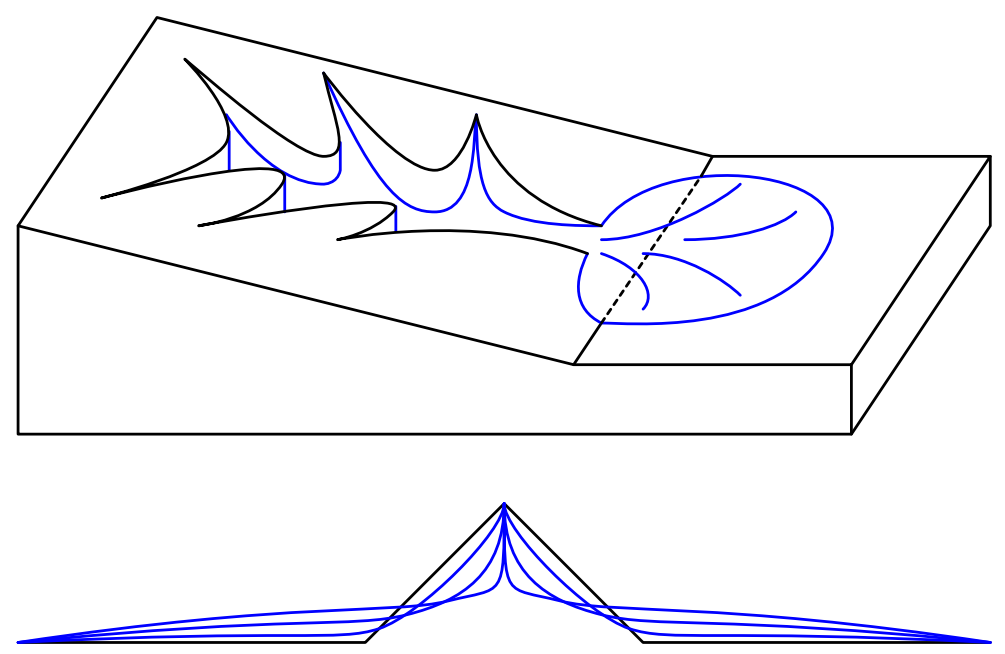

FiG. 8 - Effets de l'érosion advection.

- $d l$ une longueur élémentaire ;

$-d s=w \cdot d l$ la surface recouverte par le ruissellement associée.

Le bilan de volume d'eau s'écrit au premier ordre :

$$
\begin{gathered}
j_{e}(l) \cdot w \cdot d t+p \cdot d s \cdot d t=j_{e}(l+d l) \cdot w \cdot d t \\
j_{e}(l+d l)=j_{e}(l)+p \cdot d l
\end{gathered}
$$

La quantité maximale de sédiments transportée est délimitée par ce flux d'eau et est appelée flux de sédiments à l'équilibre [5]. Plus la pente est importante, plus cette quantité est importante :

$$
j_{s}^{e q}=j_{e} \frac{\partial h}{\partial l} k_{f}
$$

$k f$ est appelé coefficient de transport fluviatile. Le flux de sédiments tend vers le flux à l'équilibre, selon l'équation d'érosion / sédimentation suivante :

$$
\begin{gathered}
\frac{\partial h}{\partial t} \cdot d s \cdot d t=\frac{j_{s}-j_{s}^{e q}}{l_{f}} w \cdot d t \\
\frac{\partial h}{\partial t}=\frac{j_{s}-j_{s}^{e q}}{l_{f} \cdot d l}
\end{gathered}
$$

Dans le cas d'érosion $\left(j_{s}<j_{s}^{e q}\right), l_{f}$ est un paramètre d'érodabilité du substrat, qui diffère selon la nature du matériau (socle ou sédiment.) Dans le cas de sédimentation $\left(j_{s}>j_{s}^{e q}\right), l_{f}$ est petit : la rivière surchargée en sédiments 


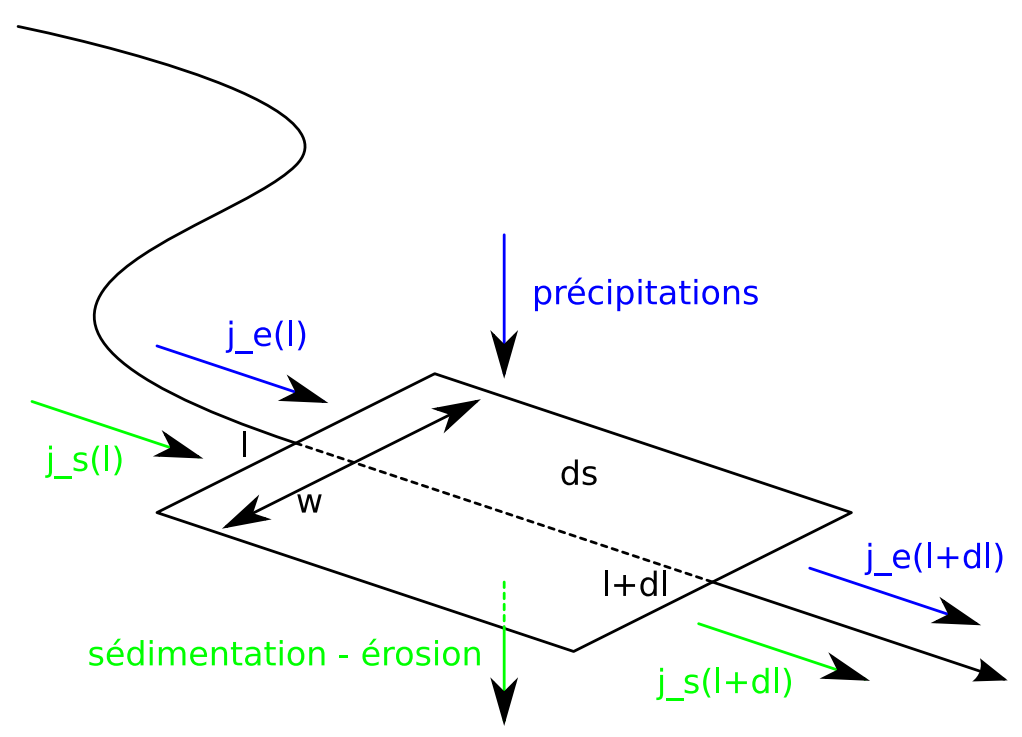

Fig. 9 - Bilans de volumes d'eau et de sédiments.

retrouve très vite son flux d'équilibre. On peut montrer que $l_{f}$ est en fait la longueur caractéristique du profil d'érosion / de dépôt des rivières (fig. 10) [3].

Le bilan de volume de sédiments s'écrit alors :

$$
\begin{gathered}
j_{s}(l) \cdot w \cdot d t+\frac{\partial h}{\partial t} \cdot d s \cdot d t=j_{s}(l+d l) \cdot w \cdot d t \\
j_{s}(l+d l)=j_{s}(l)+\frac{\partial h}{\partial t} \cdot d l
\end{gathered}
$$

\subsubsection{Discrétisation}

On utilise une méthode explicite au premier ordre. L'algorithme utilisé calcule les flux du point le plus haut jusqu'au point le plus bas de la grille considérée. La totalité de l'eau s'écoule toujours dans la plus grande pente. Dans les résultats qui suivent, la condition aux limites est l'annulation des flux de matière aux bords de la grille.

Cette méthode n'est pas exacte et peut diverger si $\delta l, \delta t, p$ et $k_{f}$ sont trop grands ou si $l_{f}$ est trop petit.

\section{Résultats}

\subsection{Paramètres d'érosion}

Cette partie contient les résultats obtenus avec le modèle précédemment décrit pour différentes valeurs du coefficient de diffusion $k_{d}$, du coefficient de 


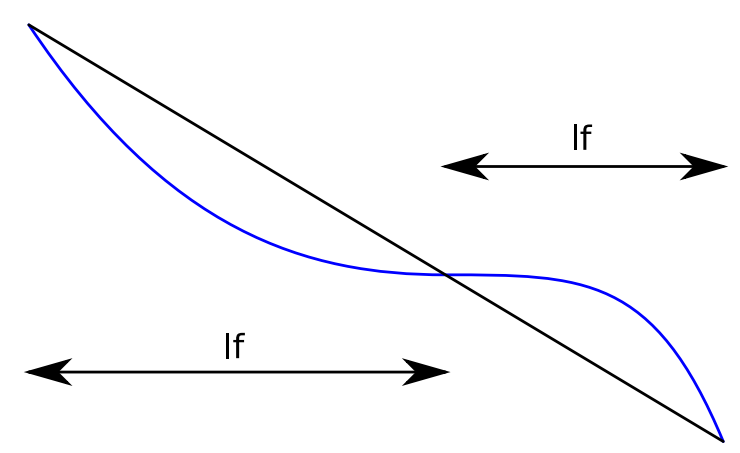

FIG. 10 - Longueurs caractéristiques.

\begin{tabular}{ll} 
paramètre & valeur \\
\hline longueur de la grille & $10 \mathrm{~km}$ \\
largeur de la grille & $5 \mathrm{~km}$ \\
taille de la maille & $100 \mathrm{~m}$ \\
durée totale & $1 \mathrm{Ma}$ \\
pas de temps & $1000 \mathrm{a}$ \\
précipitations moyennes efficaces $p$ & $1 \mathrm{~m} / \mathrm{a}$ \\
longueur caractéristique de dépôt $l_{f d e p}$ & $1 \mathrm{~km}$ \\
temps de récurrence des séismes & $3000 \mathrm{a}$ \\
profondeur de la faille & $10 \mathrm{~km}$ \\
pendage de la faille & $45^{\circ}$ \\
longueur de la faille & $20 \mathrm{~km}$ \\
glissement cosismique & $1 \mathrm{~m}$
\end{tabular}

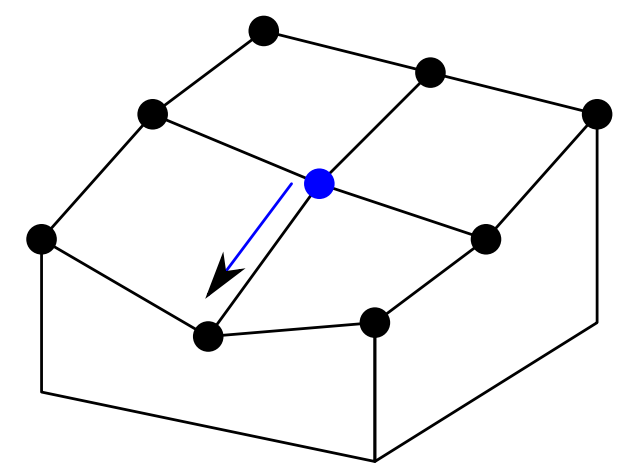

FIG. 11 - Détermination de la plus grande pente.

TAB. 1 - Paramètres fixes

transport $k_{f}$ et du paramètre d'érodabilité $l_{f}$. On ne distinguera pas ici de comportements différents pour les matériaux érodés.

Les autres paramètres sont fixées aux valeurs données dans le tableau 1 . Tous les résultats ont été obtenus à partir d'une topographie en légère pente $\left(1^{\circ}\right)$ dans le sens du pendage de la faille. Cela permet d'initier le réseau fluviatile et est cohérent avec le cas de Sparte que nous étudierons plus loin.

\subsection{Notations}

De même que l'on n'observe pas systématiquement des facettes triangulaires sur un escarpement de faille normale active, les facettes triangulaires sont un cas particulier parmi les résultats obtenus. Les différentes morphologies observées sur ces résultats peuvent être regroupées de manière quantitative en différentes catégories : 


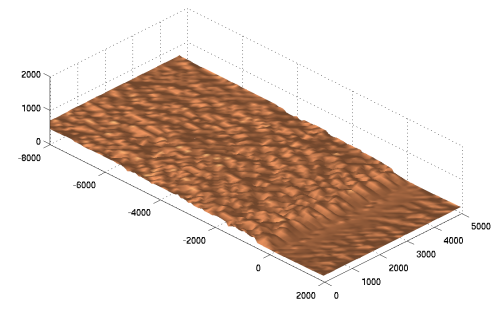

FIG. $12-\sqcup$

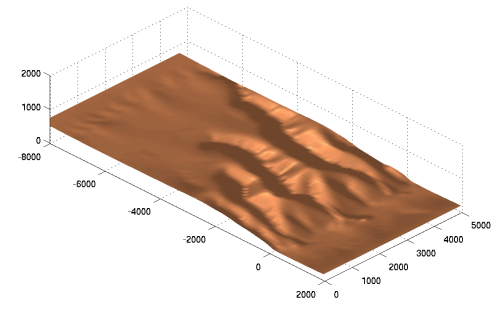

FIG. $15-\wedge$

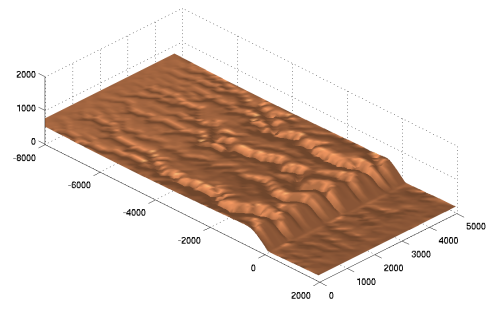

FIG. $13-\sqcap$

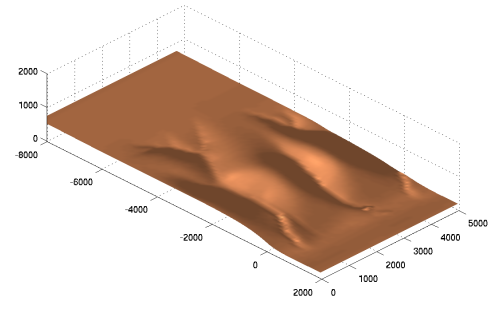

FIG. $16-\cap$

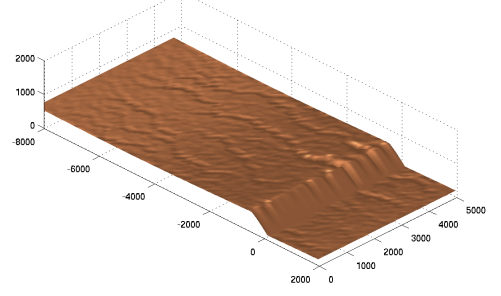

FIG. $14-=$

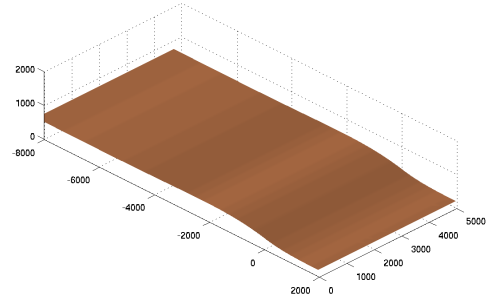

FIG. $17-\mathrm{o}$

- $\sqcup$ : escarpement très incisé, en forme de peigne (facettes plus petites que la maille);

- $\Pi$ : facettes trapézoïdales ou carrées;

$-=$ : escarpement très peu érodé;

$-\wedge:$ facettes triangulaires;

$-\cap$ : facettes très émoussées;

- o : escarpement émoussé mais pas incisé;

- - : escarpement très peu marqué (reliefs plus petits que la maille). Cette morphologie est souvent observée pour des paramètres proches de ceux qui font diverger le modèle, et peu donc être considérée comme un artefact de la discrétisation ;

- . : divergence.

Ces différentes morphologies sont le résultat d'une compétition entre diffusion et incision de l'escarpement par les rivières.

\subsection{Domaine de formation des facettes triangulaires}

Le tableau 2 montre les résultats obtenus avec le modèle pour un coefficient de transport $k_{f}=0,1$. Les différentes morphologies sont observées dans des zones relativement bien définies et la formation des facettes triangulaires 


\begin{tabular}{c|ccccccc}
$k_{d} \mid l_{f}$ & 10 & 30 & 100 & 300 & 1000 & 3000 & $10000 \mathrm{~km}$ \\
\hline 0,0003 & - & $\sqcup$ & $\sqcup$ & $\sqcup$ & $\sqcap$ & $=$ & $=$ \\
0,001 & - & $\sqcup$ & $\sqcup$ & $\sqcup$ & $\sqcap$ & $=$ & $=$ \\
0,003 & - & $\sqcup$ & $\sqcup$ & $\sqcup$ & $\sqcap$ & $=$ & $=$ \\
0,01 & - & $\sqcup$ & $\sqcup$ & $\wedge$ & $\sqcap$ & $\cap$ & 0 \\
0,03 & - & $\sqcup$ & $\wedge$ & $\wedge$ & $\cap$ & $\cap$ & 0 \\
0,1 & - & $\wedge$ & $\wedge$ & $\wedge$ & $\cap$ & 0 & 0 \\
0,3 & $\wedge$ & $\wedge$ & $\cap$ & $\cap$ & $o$ & $o$ & 0 \\
$1 \mathrm{~m}^{2} / \mathrm{a}$ & $\wedge$ & $\cap$ & $\cap$ & $\mathrm{o}$ & $\mathrm{o}$ & 0 & 0
\end{tabular}

TAB. $3-k_{f}=0.1$

\begin{tabular}{c|cccccc}
$k_{d} \mid l_{f}$ & 100 & 300 & 1000 & 3000 & 10000 & $30000 \mathrm{~km}$ \\
\hline 0,0003 & $\sqcup$ & $\sqcup$ & $\sqcup$ & $\sqcup$ & $\sqcap$ & $=$ \\
0,001 & - & $\sqcup$ & $\sqcup$ & $\sqcup$ & $\sqcap$ & $=$ \\
0,003 & - & $\sqcup$ & $\sqcup$ & $\sqcup$ & $\sqcap$ & $=$ \\
0,01 & - & $\sqcup$ & $\sqcup$ & $\sqcap$ & $\sqcap$ & $\cap$ \\
0,03 & - & - & $\wedge$ & $\wedge$ & $\sqcap$ & 0 \\
0,1 & - & - & $\wedge$ & $\cap$ & $\cap$ & 0 \\
0,3 & $\cdot$ & $\cap$ & $\wedge$ & $\cap$ & $o$ & 0 \\
$1 \mathrm{~m}^{2} / \mathrm{a}$ & $\cdot$ & $\cap$ & $\cap$ & $\circ$ & 0 & 0
\end{tabular}

TAB. $4-k_{f}=1$

semble être la conséquence d'un équilibre entre diffusion et advection. Cette zone d'équilibre peut être définie par :

$$
300 \mathrm{~m}^{3} / a<k_{d} \cdot l_{f}<1000 \mathrm{~m}^{3} / a
$$

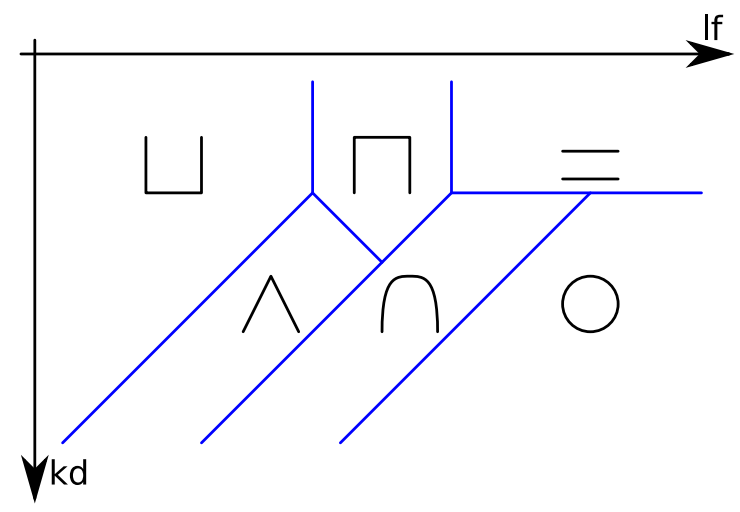

FIG. 18 - Différentes morphologies pour $k_{f}=$ cte.

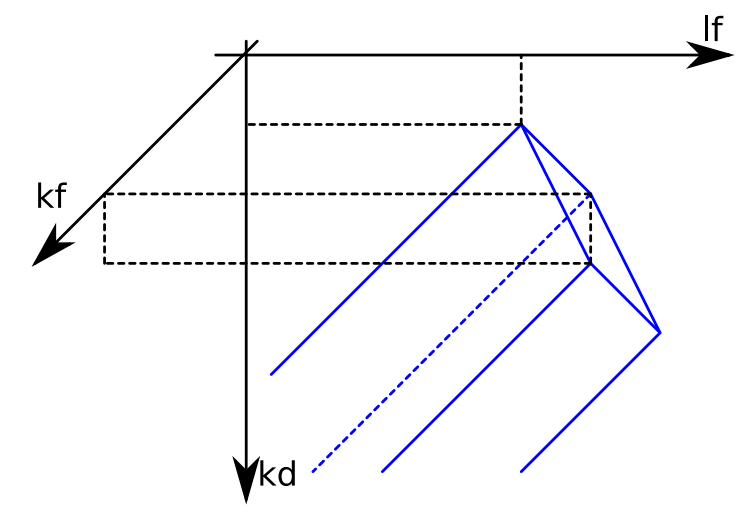

FIG. 19 - Zone de formation des facettes dans l'espace $\left(l_{f}, k_{d}, k_{f}\right)$.

Si $k_{d} \cdot l_{f}<300 m^{3} / a$, l'advection prédomine. Si $k_{d} \cdot l_{f}>1000 \mathrm{~m}^{3} / a$, la diffusion prédomine. Il existe un domaine ( $k d$ faible et $l f$ élevé) dans lequel ni la diffusion ni l'advection sont assez fortes pour éroder l'escarpement de faille (fig. 18.)

Les tableaux 3 et 4 montrent les résultats des même tests effectués pour deux valeurs différentes de $k_{f}$. On observe la même disposition des morphologies mais la zone de formation des facettes triangulaires est décalée vers les $l_{f}$ plus grandes. Compte tenu de ces résultats, on pose un nombre caractéristique de l'érosion :

$$
k_{e}=l_{f} \cdot \frac{k_{d}}{k_{f}}
$$




\begin{tabular}{c|ccccccccccccc}
$k_{d} \mid k_{f}$ & & $10^{-6}$ & & $10^{-4}$ & & $10^{-2}$ & & $10^{0}$ & & $10^{2}$ & & $10^{4}$ \\
\hline 0,003 & $=$ & $=$ & $=$ & $=$ & $\sqcap$ & $\sqcap$ & $\sqcap$ & $\sqcap$ & $\sqcap$ & $\sqcap$ & $\sqcap$ & $\sqcap$ \\
0,01 & $=$ & $=$ & $=$ & $\sqcap$ & $\sqcap$ & $\sqcap$ & $\sqcap$ & $\sqcap$ & $\sqcap$ & $\sqcap$ & $\wedge$ & $\sqcap$ \\
0,03 & $\circ$ & $\circ$ & $\circ$ & $\cap$ & $\wedge$ & $\wedge$ & $\wedge$ & $\wedge$ & $\wedge$ & $\wedge$ & $\wedge$ & $\wedge$ \\
0,1 & $\cdot$ & 0 & 0 & $\cap$ & $\wedge$ & $\wedge$ & - & - & - & - & - & - \\
$0,3 \mathrm{~m}^{2} / \mathrm{a}$ & $\cdot$ &. &. &. & - & $\wedge$ & - &. &. &. & - &.
\end{tabular}

ТАВ. $5-k_{e}=30000 m^{3} / a$

\begin{tabular}{c|cccccccc}
$k_{d} \mid k_{e}$ & 30000 & 40000 & 50000 & 60000 & 70000 & 80000 & 90000 & $100000 \mathrm{~m}^{3} / \mathrm{a}$ \\
\hline 0,003 & $\sqcap$ & $\sqcap$ & $\sqcap$ & $\sqcap$ & $\sqcup$ & $\sqcup$ & $\sqcup$ & $\sqcup$ \\
0,01 & $\wedge$ & $\wedge$ & $\wedge$ & $\wedge$ & $\sqcap$ & $\sqcap$ & $\sqcap$ & $\sqcap$ \\
0,03 & $\wedge$ & $\wedge$ & $\wedge$ & $\wedge$ & $\wedge$ & $\wedge$ & $\wedge$ & $\wedge$ \\
0,1 & $\wedge$ & $\wedge$ & $\wedge$ & $\wedge$ & $\wedge$ & $\wedge$ & $\wedge$ & $\wedge$ \\
$0,3 \mathrm{~m}^{2} / \mathrm{a}$ & - & $\wedge$ & $\wedge$ & $\wedge$ & $\wedge$ & $\wedge$ & $\wedge$ & $\wedge$
\end{tabular}

TAB. 6 - Variations de $k_{e}$ pour $k_{f}=0,01$

On peut alors décrire le domaine des facettes triangulaires dans l'espace à trois dimensions $\left(l_{f}, k_{d}, k_{f}\right)$ (fig. $19:$ )

$$
30000 \mathrm{~m}^{3} / a<k_{e}<100000 \mathrm{~m}^{3} / a
$$

Nous pouvons maintenant regarder plus précisément comment les facettes triangulaires évoluent dans ce domaine.

\subsection{Plan $k_{e}=$ cte}

On se place désormais dans le plan $k_{e}=30000 \mathrm{~m}^{3} / \mathrm{an}$. Comme le montre le tableau 5 , la forme des facettes varie fortement avec $k_{d}$, mais peu avec $k_{f}$. Cela signifie qu'au premier ordre, les effets de $k_{f}$ et de $l_{f}$ sont similaires.

On observe une nette diminution de pente des facettes avec $k_{f}$, alors que pour $k_{f}=$ cte, les pentes ne varient pas. La pente des facettes de dernière génération ne dépend donc que de $k_{f}$, ce qui est logique puisque, par définition, il s'agit des reliefs non affectés par l'érosion fluviatile.

Par ailleurs, on observe une augmentation de la taille des bassins versants avec $k_{f}$, ce qui est logique, puisqu'à $k_{e}$ fixe, $l_{f}$ augmente avec $k_{f}$. Mais il s'agit d'un effet de deuxième ordre. On fixe désormais arbitrairement $k_{f}=0,01$, valeur pour laquelle le modèle numérique semble être plus stable.

\subsection{Variations du nombre caractéristique d'érosion}

On s'intéresse désormais aux variations de $k_{e}$ au sein du domaine des facettes triangulaires, c'est à dire entre 30000 et $100000 \mathrm{~m}^{3} / \mathrm{a}$. Le tableau 6 est 
intéressant car il montre des facettes dont la pente varie de haut en bas et la forme de droite à gauche. Il permet donc de connaître les différents paramètres d'érosion à partir de la topographie actuelle d'un escarpement de faille.

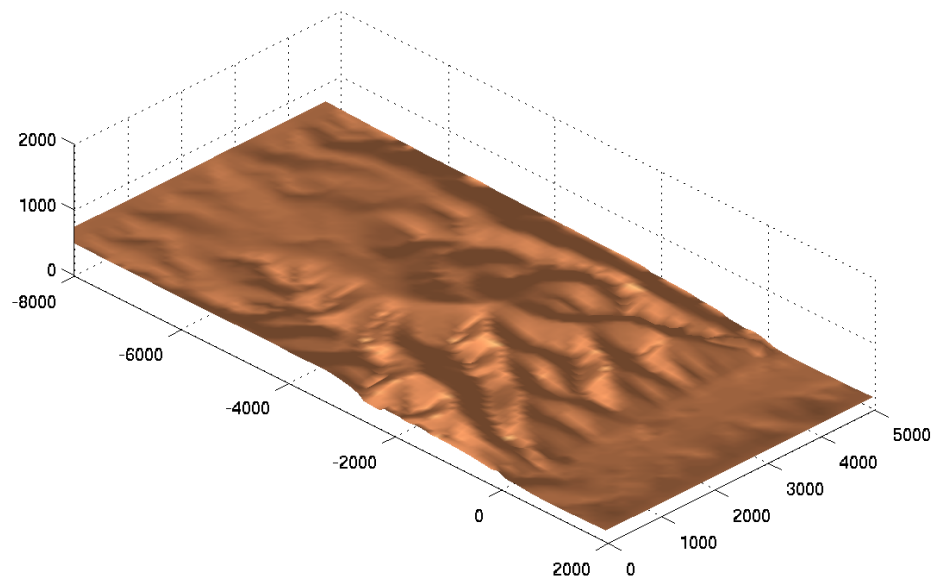

FIG. 20 - Un bel exemple de facettes imbriquées.

Cependant, les valeurs utilisées pour les précédents tests ne sont pas du tout comparables à celles qui sont observées dans la réalité à cause de l'influence de la taille de la maille et du pas de temps sur les résultats du modèle. Les valeurs des coefficients de diffusions varient plutôt entre 1 et $30 \mathrm{~m}^{2} / \mathrm{a}$, mais faire tourner le programme avec de telles valeurs aurait demandé un temps de calcul considérable, à cause de la condition de stabilité explicitée plus haut. Néanmoins, ces résultats nous permettent d'approcher de manière qualitative les phénomènes géomorphologiques observés dans la nature.

\section{Application à la faille de Sparte}

\subsection{Situation}

La faille normale de Sparte se situe à l'ouest de la ville de Sparte, dans le sud du Péloponèse. Elle est orientée NNO - SSE et fait partie du système de rifts de la mer Égée. La faille mesure $20 \mathrm{~km}$ de long pour $10 \mathrm{~km}$ de profondeur et pend à $45^{\circ}$ vers l'est. Sa vitesse actuelle est de $1 \mathrm{~mm} /$ an et le temps moyen de récurrence des séismes y est de 3000 ans environ [1].

Les reliefs associés à Sparte ont la particularité d'être en partie hérités d'une phase compressive, antérieure à l'activation de la faille normale. Il est difficile 
de distinguer la part de chacune des deux phases, compressive et extensive, dans la formation de ces reliefs. Par ailleurs, l'escarpement de faille possède une morphologie très particulière.

\subsection{Vallées en verre à vin}

Au niveau du nœud de faille, on observe un escarpement très peu érodé d'environ $10 \mathrm{~m}$ de haut, qui correspond aux séismes les plus récents [1]. Il est associé à des canyons aux parois verticales, de 10 mètres de profondeur, entre les facettes triangulaires, qui donnent aux vallées concernées une forme en verre à vin. Cela peut s'expliquer par un brusque changement climatique qui aurait eu lieu il y a 10000 ans environs. Cela correspond à la fin de la dernière glaciation.

Compte tenu de la situation géographique de Sparte, une période glaciaire se traduit par un climat très humide, qui contraste avec le climat actuel, aride, qui caractérise les interglaciaires. Cela peut se modéliser par une variation du coefficient de diffusion, ce dernier étant fortement lié à la quantité de précipitations.

On utilise les paramètres de la figure $20\left(k_{f}=0,01, l_{f}=4000, k_{d}=0,1\right)$ pour la première partie de la modélisation, puis on annule le coefficient de diffusion sur les derniers 15000 ans. Par ailleurs, à -15000 ans, on effectue un zoom sur le bas d'une vallée et on diminue la taille de la maille pour distinguer des reliefs de l'ordre de la dizaine de mètres. On observe effectivement une vallée en verre à vin (fig. 21) dont la largeur est directement liée à la taille de la maille.

\subsection{Ruptures de pentes}

Les facettes de Sparte présentent un nette rupture de pente à environ 450 mètres [1] de hauteur au-dessus du bassin sédimentaire (fig. 23.) Il s'agit à nouveau d'un changement climatique. Ainsi, on peut considérer que la totalité de l'érosion diffusion sur l'escarpement de Sparte se fait pendant les glaciations.

La figure 22 représente le résultat en coupe de la modélisation de l'effet des quatre derniers interglaciaires [8] :

- 540 - $480 \mathrm{ka}:$ Günz - Mindel ;

- 430 - $240 \mathrm{ka}$ : Mindel - Riss;

- 180 - 120 ka : Riss - Würm;

- 15 - 0 ka : interglaciaire actuel.

On observe plusieurs ruptures de pentes liées à la fin de chaque période glaciaire. À nouveau, ces modélisations ne sont pas quantitatives.

\section{Conclusion}

Ce modèle numérique de formation de facettes triangulaires est donc applicable à des cas réels, comme celui de la faille de Sparte. Il permet de relier l'évolution climatique d'une région à son histoire tectonique par l'intermédiaire des reliefs observés sur un escarpement de faille. Cependant, il est encore nettement incomplet. 

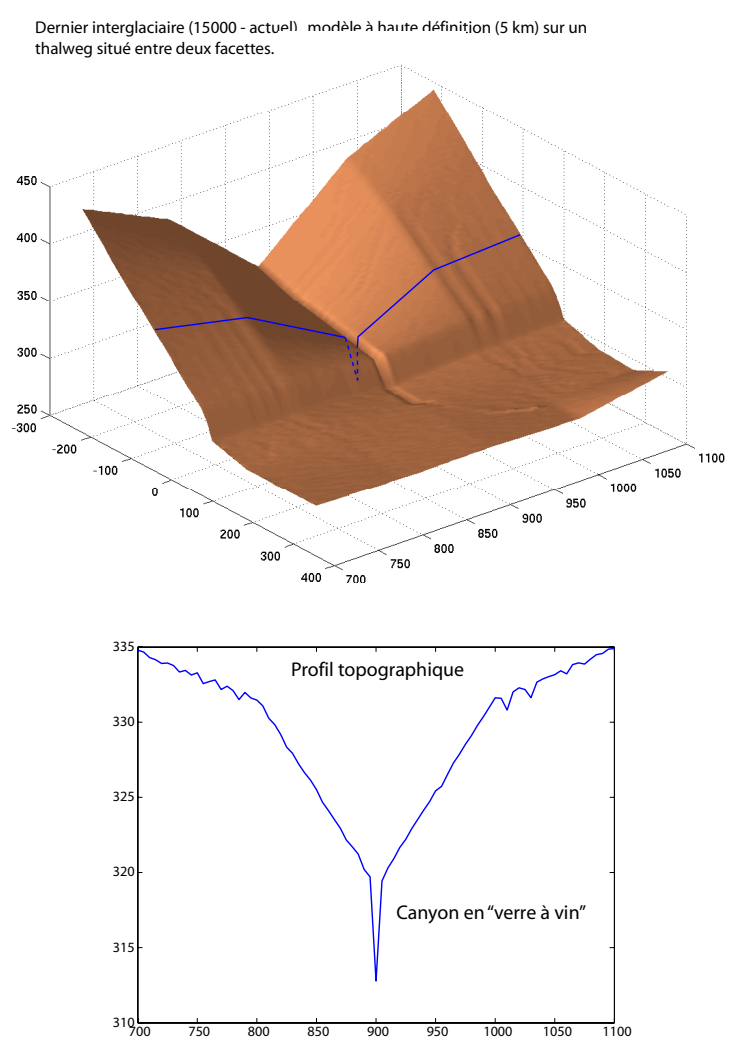

Fig. 21 - Modélisation d'une vallée en verre à vin image de Carole Petit.
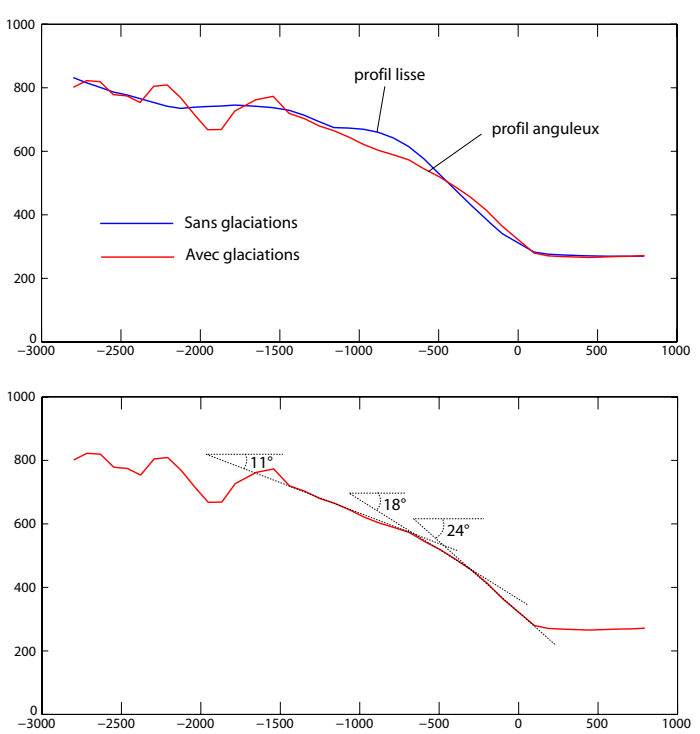

FIG. 22 - Modélisation des changements climatiques image de Carole Petit

Tout d'abord, il manque cruellement de données de terrain. Plusieurs des paramètres du modèle, comme le coefficient de diffusion, pourraient être déterminés, ou au moins estimés. D'autres tests sont à effectuer, notamment en ce qui concerne les paramètres tectoniques. Le programme n'a jamais tourné avec des paramètres proches de la réalité. L'espacement des rivières lors de l'initialisation du réseau fluviatile et donc la largeur des facettes de première génération n'est pas contrainte par le programme. Les rivières ont une largeur systématiquement égale à la taille de la maille utilisée.

Mais malgré ces nombreuses lacunes, ce stage m'a apporté des notions de géomorphologie et d'informatique, ainsi qu'une première approche du travail en laboratoire de recherche. 


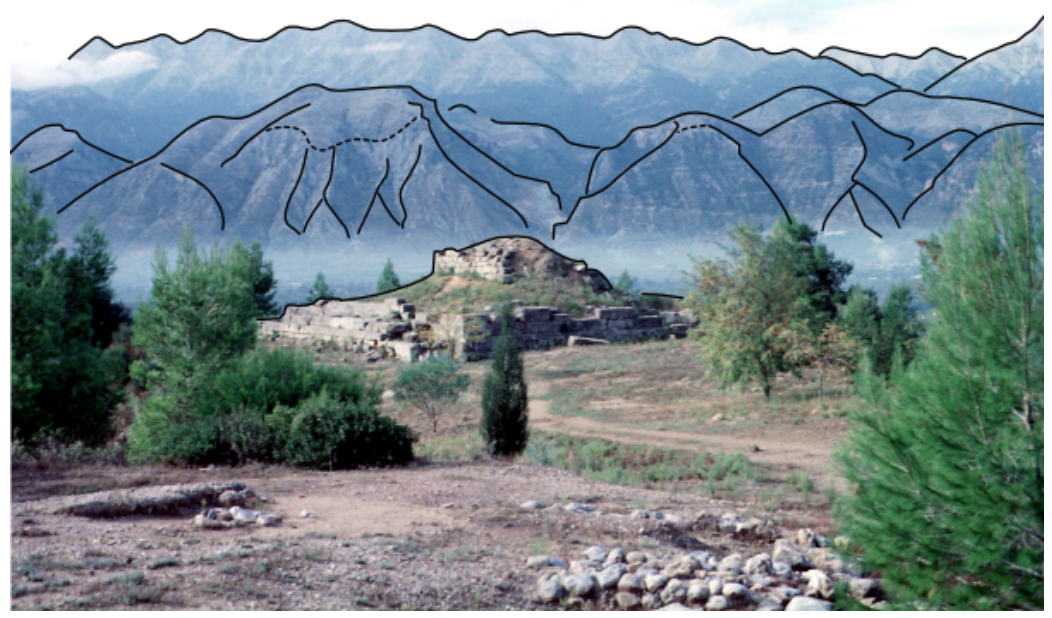

FIG. 23 - Facettes triangulaires de Sparte - photo originelle de John Hansen [6]

\section{Références}

[1] R. Armijo, H. Lyon-Caen et D. Papanastassiou, A possible normal-fault rupture for the 464BC Sparta earthquake, Nature, Vol 351, 137-139, 1991.

[2] C.A. Cotton, Tectonic scarps and fault valleys, Bullutin of the Society of Geology of America, Vol. 61, 717-758, 1950.

[3] H. Kooi et C. Beaumont, Escarpment evolution on high-elevation rifted margins : Insights derived from a surface processes model that combines diffusion, advection, and reaction, Journal of Geophysical Research, Vol. 99, 12191-12209, 1994.

[4] Y. Okada, Surface deformation due to shear and tensile faults in a halfspace, Bulletin of the Seismological Society of America, Vol. 75, 1135-1154, 1985.

[5] P. van der Beek, M. A. Summerfield, J. Braun, R. W. Brown et A. Fleming, Modeling postbreakup landscape development and denudational history across the southeast African (Drakensberg Escarpment) margin, Journal of Geophysical Research, Vol. 107, 2351, 2002.

[6] Stoa Image Gallery, www.stoa.org/gallery/awmcsparta/MenelaionAndView

[7] Catalogue Nasa Visible Earth, visibleearth.nasa.gov

[8] Encyclopédie Wikipédia, fr.wikipedia.org/wiki/Glaciation

Ce document est libre de droits. 